\title{
Philosophiques
}

\section{Une bonne mort ? (Blanchot, Derrida)}

\section{Cosmin Toma}

Volume 47, numéro 2, automne 2020

\section{Derrida en cours}

URI : https://id.erudit.org/iderudit/1075130ar

DOI : https://doi.org/10.7202/1075130ar

Aller au sommaire du numéro

Éditeur(s)

Société de philosophie du Québec

ISSN

0316-2923 (imprimé)

1492-1391 (numérique)

Découvrir la revue

Citer cet article

Toma, C. (2020). Une bonne mort ? (Blanchot, Derrida). Philosophiques, 47(2), 393-415. https://doi.org/10.7202/1075130ar

\section{Résumé de l'article}

Le Séminaire La peine de mort de Jacques Derrida est hanté, depuis ses marges, par la question de l'euthanasie. Si Derrida y fait allusion à plusieurs reprises au fil des séances, il ne la laisse pas moins en suspens, nous indiquant par là qu'il s'agit d'une problématique à part entière. Or dans "Survivre ", texte de 1979 sur L'Arrêt de mort de Maurice Blanchot, Derrida évoquait déjà le " double bind qui fait de toute mort un crime ", déjouant ainsi cette distinction, censée garantir la frontière éthique entre l'euthanasie et la peine capitale. À travers une relecture de L'Arrêt de mort qui prend appui sur le Séminaire La peine de mort, cet article analyse la porosité des différentes déclinaisons de la mort lorsqu'elle est pensée comme crime, à commencer par l'arrêt, la peine et la sentence (ou « death sentence »). 


\title{
Une bonne mort? (Blanchot, Derrida)
}

\author{
COSMIN TOMA \\ Chercheur postdoctoral \\ Faculty of Medieval and Modern Languages \\ University of Oxford
}

\begin{abstract}
RÉSUMÉ. - Le Séminaire La peine de mort de Jacques Derrida est hanté, depuis ses marges, par la question de l'euthanasie. Si Derrida y fait allusion à plusieurs reprises au fil des séances, il ne la laisse pas moins en suspens, nous indiquant par là qu'il s'agit d'une problématique à part entière. Or dans «Survivre», texte de 1979 sur L'Arrêt de mort de Maurice Blanchot, Derrida évoquait déjà le «double bind qui fait de toute mort un crime», déjouant ainsi cette distinction, censée garantir la frontière éthique entre l'euthanasie et la peine capitale. À travers une relecture de L'Arrêt de mort qui prend appui sur le Séminaire La peine de mort, cet article analyse la porosité des différentes déclinaisons de la mort lorsqu'elle est pensée comme crime, à commencer par l'arrêt, la peine et la sentence (ou «death sentence»).
\end{abstract}

ABSTRACT. - Jacques Derrida's Death Penalty seminar is haunted, from its margins, by euthanasia. Yet even as he alludes to this question throughout the seminar, he puts it on hold, no doubt because it calls for a standalone analysis. In "Living On," however, Derrida's 1979 reading of Maurice Blanchot's Death Sentence (L'Arrêt de mort), he refers to "a 'double bind' that makes every death a crime," thus subverting this very distinction, which is meant to ensure the hard ethical border between euthanasia and capital punishment. Through a rereading of Blanchot's Death Sentence that takes its cues from Derrida's Death Penalty seminar, this article analyses the porous nature of death's various instantiations when it is conceived as a crime, beginning with the "arrêt" (simultaneously a stop and a judgment), the penalty and the sentence.

\section{Death Sentence}

Tel est le titre retenu par Lydia Davis pour sa traduction anglaise, parue en 1986, de L'Arrêt de mort de Maurice Blanchot. Synonyme de la «death penalty" sans l'être tout à fait, "death sentence» nomme l'instant où la peine de mort est prononcée. Traduite littéralement, il s'agit d'une sentence mortelle, peut-être proférée par la mort même. Ce point d'intraductibilité, Jacques Derrida le relève dès 1979 dans "Survivre" », une lecture minutieuse de L'Arrêt de mort dont la première version paraîtra en anglais dans un ouvrage collectif consacré à la critique littéraire, Deconstruction and Criticism. Dans ce texte bifide, doublé d'un commentaire du "Triumph of Life» de Percy Bysshe Shelley, Derrida s'adresse directement à son traducteur, le mettant au défi de rendre justice à certaines tournures réputées intraduisibles, à commencer par «l'arrêt de mort». En effet, si en français l'arrêt

1. Jacques Derrida, «Survivre», dans Parages, Paris, Galilée, 1986, p. 117-218. 
désigne une "[d]écision rendue par une cour souveraine", comme le rappelle le Littré, qui donne pour exemple (et c'est tout sauf un hasard) «un arrêt de mort $»^{2}$, il renvoie toujours, a minima, à ce qui s'arrête, selon un principe idiomatique et autoréflexif qui, lorsqu'on l'applique au titre de Blanchot, "interdit, empêche, inhibe, arrête une traduction de L'arrêt de mort ${ }^{3}$ ". La "sentence» anglaise, quant à elle, et pour ne s'en tenir qu'à ses définitions les plus courantes, est à la fois une condamnation et un speech act ou, plus simplement, une phrase.

Non seulement cette attention prêtée à l'intraduisible multiplicité des langues est-elle un aspect remarquable de l'analyse derridienne de L'Arrêt de mort, le récit lui-même (mais en est-ce $u^{4}$ ?) y fait allusion à plusieurs reprises. Dès son inscription initiale en français - et tout en sachant que cette primauté chronologique est, comme on le verra, infiniment moins sûre qu'il n'y paraît —, il s'ouvre ainsi à d'autres idiomes, à d'autres formulations ou états contrefactuels. Autrement dit, l'expression "l'arrêt de mort» contient en germe «la phrase de la mort», "la décision de la mort», «le dicton de la mort», "la loi de la mort», «la fin de la mort», "le sursaut de la mort ", "l'interruption de la mort», "la sentence de la mort», ainsi que, de manière encore plus immédiate, "la peine de mort». Nous avons donc affaire à une chaîne lexicale d'une complexité et d'une diversité redoutables, suspendue à la mort même ou, à tout le moins, à la question philosophicolittéraire de la mort, qui porte toujours plus d'un nom, dans plus d'un idiome ( Mon gosier de métal parle toutes les langues ${ }^{5}$ ", pour citer l'horloge funeste des Fleurs du mal de Baudelaire).

Deux décennies plus tard, lorsque Derrida abordera à nouveau le problème de la mort par l'entremise de la peine capitale, il réitérera cette multiplicité en affirmant qu' «il n'est pas sûr qu'il y ait un problème de la peine de mort, un seul et même problème identifiable sous ce nom. Ce nom, ce titre, la peine de mort, dissimule peut-être une multiplicité non unifiable de concepts et de questions. Nous ne devrions jamais l'exclure ${ }^{6}$.» C'est l'une des principales hantises du Séminaire La peine de mort, qui souligne sans relâche la difficulté de cerner le propre de la peine de mort, comme si quelque chose en empêchait la saisie ou la conceptualisation. Sur cette question viennent ainsi se greffer celles, non moins poreuses, du meurtre, du suicide,

2. Le Littré, [en ligne: https://www.littre.org]. Consulté le 27 juin 2019.

3. Derrida, "Survivre», p. 152.

4. «Un récit? Non, pas de récit, plus jamais.» (Maurice Blanchot, La Folie du jour, Montpellier, Fata Morgana, 1973, p. 18.) Cette proposition s'applique également à L'Arrêt de mort, puisque Blanchot lui a retiré cette appellation de genre, tout en supprimant les deux dernières pages de la première version, lors de sa réédition en 1971.

5. Charles Baudelaire, "L'Horloge», dans Euvres complètes, t. I, Paris, Gallimard, 1975 , p. 81.

6. Derrida, Séminaire La peine de mort. Volume II (2000-2001), Paris, Galilée, 2015, p. 39. 
de la guerre, de l'avortement, de la littérature, du politique et du juridique. À quoi il importe d'ajouter: la question de l'euthanasie, qui inquiète le séminaire de Derrida depuis ses marges et qui se situe au cœur même de L'Arrêt de mort de Blanchot, d'où l'importance d'emprunter, comme je le ferai ici, un long détour intertextuel, dans l'espoir de rendre justice à la multiplicité qui est en jeu ici, faisant signe vers la différance même de la mort.

Cette problématique peut également se formuler comme suit: en quoi consiste une "bonne mort", comme le voudrait, étymologiquement, l'euthanasie (eú + thánatos) ? Dans quelles circonstances, à quelles conditions la mort a-t-elle le potentiel de devenir «bonne» ou, lorsqu'on la conjugue au problème de la peine de mort, «juste » ? Cette question est peut-être même à l'origine du différend entre Blanchot et Derrida, entre l'auteur de «La littérature et le droit à la mort» et celui qui nous aura enjoint à "[p]référe[r] toujours la vie ", à "affirme[r] sans cesse la survie " ${ }^{7}$, même si l'on aurait assurément tort de réduire cette différence à un antagonisme complaisant tel que «Blanchot, la mort» et "Derrida, la vie », ne serait-ce qu'en raison de ce troisième terme - la survie ${ }^{8}$ - qui sous-tend, dès 1979 , la lecture que Derrida nous livre de L'Arrêt de mort. Dans ces parages, il y a aussi, bien entendu, La Folie du jour, ainsi que L'Instant de ma mort, récits indécidables commentés, eux aussi, par le philosophe et que Ginette Michaud nous aura exemplairement aidés à entendre, au même titre que leurs exégèses derridiennes ${ }^{9}$. J'aimerais donc à mon tour revenir sur ce dialogue (en admettant que ce soit là le mot juste), à mi-chemin entre le Séminaire La peine de mort de Derrida et L'Arrêt de mort de Blanchot, entre la philosophie et la littérature, afin d'en prolonger les résonances à partir de la question de l'euthanasie, d'abord et avant tout.

Car si celle-ci est nommée à plusieurs reprises au fil des séances, Derrida ne la laisse pas moins en suspens, nous indiquant par là qu'il s'agit d'une aporie à part entière dont il traitera, peut-être, ailleurs. Or dans la mesure où «Survivre» évoquait déjà le «double bind qui fait de toute mort un crime ${ }^{10}$ ", cette distinction entre mort et meurtre, censée garantir la frontière éthique entre l'euthanasie et la peine capitale, s'en voit profondément inquiétée. À travers une relecture de L'Arrêt de mort qui prend appui sur le Séminaire La peine de mort, je vais donc creuser plus avant la porosité littéraire, pourrait-on dire, c'est-à-dire aporétique — des différentes décli-

7. Cité par René Major dans Derrida pour les temps à venir, Paris, Stock, 2007, p. 508.

8. Disant bien la subsistance (ou survie) de cette question d'un bout à l'autre du corpus derridien, un autre séminaire aurait pu être convoqué ici : La vie la mort (Paris, Seuil, 2019), ne serait-ce qu'en raison de son recours, encore une fois, à Blanchot et tout particulièrement au Pas au-delà, ce titre qui dit à lui seul le mouvement de transgression qui s'opère (sans la conjonction «et») entre vie-mort/mort-vie, sans jamais s'arrêter sur l'un ou l'autre de ces deux termes.

9. Ginette Michaud, Tenir au secret (Derrida, Blanchot), Paris, Galilée, 2006.

10. Derrida, «Survivre», p. 163. 
naisons de la mort lorsqu'elle est pensée comme crime, à commencer par l'arrêt, la peine et la sentence (ou "death sentence»).

Non moins que l'arrêt de mort, la peine de mort, dite aussi «peine capitale », est un nom ou un titre qui ne se laisse pas aisément circonscrire. Pourtant, "la peine de mort» est aussi un nom commun unique, doté d'une signature qui lui est propre. Il s'agit plus précisément d'un nom français, à l'image de celui de Joseph-Ignace Guillotin. Cela, Derrida le rappelle à plusieurs reprises dans le Séminaire La peine de mort, soulignant, d'une part, le caractère foncièrement national de l'histoire de la peine de mort — après tout, l'État-nation, même celui qui, à l'image de la Ve République française, ou du Canada, a fini par abolir la peine de mort, se réserve encore et toujours le droit de mettre à mort ses ennemis (pour se défendre en temps de guerre, d'abord et avant tout $\left.{ }^{11}\right)$ — et, d'autre part, la tendance internationale, confirmée par les recommandations de l'ONU en 2007, 2008, 2010, 2012 et 2014, à considérer la peine capitale comme une chose du passé, un anachronisme déplorable que des pays tels que la Turquie de Recep Tayyip Erdoğan se disent pourtant prêts à rétablir dans un avenir plus ou moins proche.

Le syntagme ou concept de peine de mort n'a donc pas le même sens d'un pays à l'autre, d'une langue à l'autre, d'une époque à l'autre. En roumain, par exemple, "peine de mort » se dit «pedeapsa cu moartea ", expression étymologiquement métissée en ce que pedeapsa provient du grec epedepse, aoriste de pedevo, qui renvoie à la paideia, c'est-à-dire à l'instruction, tandis que moartea, sans surprise, puise ses origines dans le latin. L'étymologie du français "peine» est en apparence moins retorse, puisque ce mot découle, en dernière analyse, de la poiné, autre terme grec qui signifie le châtiment et la délivrance, ainsi que la rançon. Entre la peine comme paideia ou leçon (Derrida s'y réfère dès l'introduction du séminaire, en évoquant les sophronistères de la Grèce antique) et la peine comme poiné ou châtiment, l'écart est de taille. De même, distinguer entre la «death penalty » et la "death sentence», plutôt qu'entre "la peine de mort» et "l'arrêt de mort ", suppose une certaine phraséologie: celle du juge prononçant la sentence, justement, comme si la question tout entière pouvait se résumer à ce moment performatif, décisif et critique (dans tous les sens de ce terme) où la phrase devient couperet.

Bien entendu, on ne saurait réduire la peine capitale à un vocable - au contraire, elle fait violence au langage en le débordant de toute part —, mais son appellation mérite d'être prise au sérieux au même titre que la «chose» même. Nous voici donc déjà dans les parages de la pensée de Blanchot, et tout particulièrement de «La littérature et le droit à la mort », qui esquisse

11. Inutile d'aller bien loin: le cas du Canada témoigne de cette ambiguité de manière exemplaire. Bien qu'abolie de facto en 1963, la peine de mort demeura applicable de jure en cas de mutinerie jusqu'en 1998. 
un trait d'union en apparence indélébile entre le langage, la mort et la Terreur, et que Derrida commente explicitement dans le Séminaire La peine de mort. Dans la séance d'introduction, il semble pourtant se méfier de ce texte, évoquant, non sans ironie, "de grandes veines comme "la littérature et la mort", "la littérature et le droit à la mort" 12 ", comme s'il y avait là un lien cousu de fil blanc, comme si Blanchot était trop proche de la peine de mort pour qu'on puisse en tirer une réflexion qui aille au-delà des clichés habituels.

Trois séances plus tard, le Séminaire La Peine de mort y consacrera tout de même un important développement. En guise de préambule, Derrida souligne l'ambiguité du texte de Blanchot, qui peut aisément se lire, dit-il, "comme le terrifiant document d'une certaine époque de la littérature française, très française, et de la meilleure, de la plus fascinante, de la plus fascinée, mais aussi de la plus équivoque pensée politique de la littérature ${ }^{13}{ }^{1}$. Il marque ainsi une réserve, aussi élogieuse fût-elle, à l'endroit de ce que « La littérature et le droit à la mort» peut avoir d'absolutiste et de sanglant, comme si la littérature exigeait non seulement que l'on s'expose à la mort mais aussi qu'on la donne à autrui. Or tout en reconnaissant la validité d'une lecture qui se placerait résolument du côté de la mort, voire du meurtre, Derrida annonce qu'il interprétera plutôt «ce document [...] comme le contre-point, mais au nom de la littérature même, de ce droit inviolable à la vie et comme le contre-pied hegeliano-mallarméen de l'abolitionnisme hugolien ${ }^{14}$ ». L'invocation de «La littérature et le droit à la mort» dans le Séminaire La peine de mort a donc une valeur stratégique, d'abord et avant tout, puisque Derrida ne saurait se contenter de l'argumentaire théologisant, voire néo-christique d'un Victor Hugo, qui repose sur des présupposés métaphysiques dont Blanchot se départit volontiers.

Contre le sacre romantique de la vie, qui est toujours suspendu à quelque théologème et donc à cela même qui a historiquement servi de justification à la peine capitale, "La littérature et le droit à la mort » affirme la neutralité toute-puissante de la mort, anonyme et vide de sens à tel point qu'elle passe presque inaperçue. De manière plus prosaïque, Derrida met également en relief le contraste entre Blanchot et Hugo à travers la datation, qui joue, comme on le verra, un rôle essentiel dans tout ce débat autour de la peine de mort. Le philosophe nous rappelle en effet que la première parution de «La littérature et le droit à la mort ", dans la revue Critique en 1948, coïncide plus ou moins avec celle de la Déclaration universelle des droits de l'homme, adoptée la même année, mais aussi et surtout avec le centenaire d'un célèbre discours abolitionniste de Victor Hugo, commenté dans la pre-

12. Derrida, Séminaire La peine de mort. Volume I (1999-2000), Paris, Galilée, 2012, p. 59.

13. Ibid., p. 164.

14. Ibid. 
mière partie de la séance du 19 janvier 2000. Cela dit, il n'y a pas lieu de prendre ces cent ans de différence à la lettre, car ce sont deux quasi contemporains de Hugo - Hegel et Mallarmé - qui soufflent à Blanchot « la force négative ou annihilante de la nomination, le lien essentiel entre le langage et la mort, le langage et le meurtre, le langage qui anéantit l'existence de la chose $^{15}$ ", radicalisant ainsi le problème des rapports entre mort et langage en posant rien de moins que leur consubstantialité. Qui plus est, si Blanchot tire le motif immémorial de la vie comme condamnation à mort du côté de l'altérité, il le fait à rebours de l'éthique levinasienne. En effet, le postulat de "La littérature et le droit à la mort» est que nous sommes condamnés à commettre un meurtre, fût-il d'ordre strictement symbolique, chaque fois que nous nommons quelque chose ou quelqu'un, appréhendant ainsi les étants à partir de leur disparition passée ou à venir. C'est là une constante chez Blanchot, ainsi que Derrida le fait remarquer dans "Survivre", en citant cet extrait de L'Écriture du désastre: "Il y a mort et meurtre (mots que je mets au défi de distinguer sérieusement et qu'il faut cependant séparer); de cette mort et de ce meurtre, c'est un “on” impersonnel, inactif et irresponsable, qui a à répondre ${ }^{16} »$.

Il faut séparer ces deux vocables, dit Blanchot - sans doute pour des raisons éthiques - et pourtant, ils lui semblent à bien des égards indissociables. Cette complicité en apparence indissoluble entre la mort et le meurtre a pour Blanchot un répondant historique: la révolution, ce moment où "[c]haque homme cesse d'être un individu travaillant à une tâche déterminée, agissant ici et seulement maintenant», car "la liberté universelle [...] ne connaît ni ailleurs ni demain, ni travail ni oeuvre ${ }^{17}$ ». Pour l'auteur de La Part $d u f e u$ - en tout cas dans ce contexte d'après-guerre, vingt ans avant Mai 68, très exactement —, l'état révolutionnaire ne s'accomplit que sous la Terreur, qui serait l'aboutissement du célèbre cri «la liberté ou la mort». C'est d'ailleurs à ce moment de «La littérature et le droit à la mort » que Blanchot élucide le titre de son texte: "Chaque citoyen a pour ainsi dire droit à la mort: la mort n'est pas sa condamnation, c'est l'essence de son droit; il n'est pas supprimé comme coupable, mais il a besoin de la mort pour s'affirmer citoyen et c'est dans la disparition de la mort que la liberté le fait naître ${ }^{18}$." Proposition d'une complexité redoutable, qui marque au passage une réticence, aussi partielle et paradoxale fût-elle, à l'endroit de la peine de mort: n'étant plus une "condamnation », la mort s'avère le fondement de toute liberté, comme s'il fallait que l'existence même fût mise en jeu pour que l'on soit non seulement quitte du péché originel (dans l'espace

15. Ibid., p. 165.

16. Blanchot, L'Écriture du désastre, Paris, Gallimard, 1980, p. 115-116.

17. Blanchot, "La littérature et le droit à la mort ", La Part du feu, Paris, Gallimard, 1949, p. 309. L'italique est dans le texte.

18. Ibid. L'italique est dans le texte. 
métaphysique judéo-chrétien, telle est en effet la condamnation à mort par excellence) mais aussi et surtout du couplage traditionnel innocence/culpabilité, déjouant ainsi la logique de la punition dans son ensemble.

Tout se passe comme si Blanchot cherchait à neutraliser la peine de mort en la devançant et en lui retirant son caractère exceptionnel, geste inattendu en ce que la révolution - et, à plus forte raison, la Révolution française et sa Terreur - est volontiers conçue en termes d'état (pour ne pas dire d'État) d'exception. Par ailleurs, cette neutralisation est aussi une anonymisation: «on » meurt comme «on » tue au nom de la révolution et l'État qui commande ces exécutions représente non pas tel ou tel monarque, mais tous les Français. La Terreur serait ainsi non moins dépersonnalisée et dépersonnalisante que la guillotine, cet outil inanimé qui en porte l'exigence meurtrière et qui témoigne à sa manière du devenir-commun du nom propre. Qui plus est, dans la mesure où cet anonymat est aussi, pour Blanchot, une condition de possibilité de la littérature, c'est tout l'espace littéraire qui se voit désormais complice du couperet. Derrida peut ainsi parler, à juste titre, de «l'alliance littérale de la littérature avec la Terreur comme machine à guillotiner ${ }^{19} »$ chez Blanchot.

Cet anonymat terrible où l'on s'expose à l'immédiat de la mort afin de faire advenir la liberté absolue — c'est-à-dire, pour Blanchot, la littérature - serait exemplifié par la figure du marquis de Sade. Dans la mesure où toute exemplification menace de déstabiliser le schéma collectif, antihéroïque et républicain de "La littérature et le droit à la mort ", cela est fort curieux. Toujours est-il qu'il y a des raisons biographiques d'accorder ce privilège au Divin Marquis, car il est de ces aristocrates devenus révolutionnaires qui, après avoir été arbitrairement condamnés à mort sous la Terreur, y échappèrent (pour des raisons, du reste, obscures). De plus, les longues années passées en prison, où il rédige clandestinement ses romans les moins publiables, font de lui un martyr rétrospectif de la liberté d'expression littéraire $^{20}$ et donc le plus moderne des écrivains français du XviII ${ }^{\mathrm{e}}$ siècle, celui qui «se reconnaît dans la Révolution [...] parce qu'elle est le temps où la littérature se fait histoire ${ }^{21}$. Enfin, comme le souligne Derrida, le nom de

19. Derrida, Séminaire La peine de mort, I, p. 165-166.

20. «La littérature est une invention moderne, elle s'inscrit dans des conventions et des institutions qui, pour n'en retenir que ce trait, lui assurent en principe le droit de tout dire » (Derrida, Passions, Paris, Galilée, 1993, p. 64-65.) Comme le rappelle Peggy Kamuf, ce droit est aussi "the right to say nothing, or rather the right and even the obligation not to respond to a demand to know, to divulge the hidden or inapparent, to make public what is secret" ( "Beginning with Literature», in Kelly Oliver et Stephanie Straub (dir.), Deconstructing the Death Penalty: Derrida's Seminars and the New Abolitionism, New York, Fordham University Press, 2018, p. 15-16.)

21. Blanchot, "La littérature et le droit à la mort», p. 323-324. Les italiques sont dans le texte. Derrida ajoute: "Il dit la même chose que Hugo, mais le contraire, n'est-ce pas! À savoir que l'écrivain fait l'histoire [...]. Il dit la même chose, mais il dit le contraire, puisque Hugo le dit du côté de la vie, et Blanchot de la mort» (Séminaire La peine de mort, I, p. 166.) 
Sade désigne aussi, et ce n'est pas anodin, "une cruauté absolue» et "une perversion radicale ${ }^{22}$, qui vont de pair avec l'imaginaire de la peine de mort, ainsi qu'avec les actes impardonnables censés la mériter. Or Blanchot nous dépeint, quant à lui, un Sade plutôt serein, «celui pour qui la mort est la plus grande passion et la dernière des platitudes, qui coupe les têtes comme on coupe une tête de chou, avec une indifférence si grande que rien n'est plus irréel que la mort qu'il donne, et cependant personne n'a senti plus vivement que la souveraineté était dans la mort, que la liberté était mort ${ }^{23}$ ". Si l'on a raison de parler ici de "cruauté » et de "perversion", elles sont nées de l'apathie la plus totale, d'un "faire-comme-si-de-rien-n'était» qui se substitue à ce «faire-semblant » que l'on a parfois proposé comme définition de l'art et de la littérature.

Derrida ne pense pas qu'il faille lire dans ces propos un «éloge de la peine de mort comme telle", tout en ajoutant que "même si on ne peut y voir une thèse assumée par lui, mais avant tout une analyse de ce qu'est ou de ce que tente d'être la littérature ", la «logique ${ }^{24}$ de Blanchot comporte des risques. Elle témoigne, ainsi que Derrida ne cesse de le souligner, d'une tentation qui laisse «à Blanchot la possibilité de dire que cette tentation, il l'analyse, il la décrit, il la constate, il en pense le mouvement essentiel sans y céder lui-même de part en part ${ }^{25}$ ", mais qui, à l'instar de l'œuvre sadienne, n'exclut pas la perversion, c'est-à-dire la réversibilité chiasmatique des deux termes ci-déployés, «la littérature» et «la peine de mort». Derrida préfère, in fine, y discerner une tentation demeurée tentation. Et pourtant (mais cela est moins contradictoire qu'on pourrait le penser), la signature blanchotienne est ici des plus équivoques, sans doute à dessein, parce qu'il s'agit d'une contresignature littéraire. En effet, il n'est pas exclu que la liberté littéraire nécessite très précisément d'aller jusqu'au bout de cette tentation thanatologique pour s'accomplir. C'est en tout cas ce que les romans et récits de Blanchot, dont L'Arrêt de mort, semblent indiquer, pour peu que cette pulsion de mort, si l'on peut dire, ne dépasse pas l'état de «fantasme » (mais un fantasme n'est-il pas toujours plus qu'un fantasme?).

Au-delà de sa volonté de tenir la chose littéraire en respect en lisant «La littérature et le droit à la mort » sous son jour le plus favorable (n'oublions pas qu'en 2000, Blanchot est toujours vivant), Derrida met le doigt sur un problème qui ne cesse de se poser dans certains des écrits d'aprèsguerre de Blanchot, et qui sera partiellement «résolu» par le recours de plus en plus insistant au neutre à partir de la fin des années 1950: la question du sacrifice. En effet, le sacrifice, en un sens qui est tout sauf étranger à Hegel, suppose que la mort puisse être relevée, qu'elle ait un sens autre que l'infini

22. Ibid.

23. Blanchot, «La littérature et le droit à la mort », p. 311. Les italiques sont dans le texte.

24. Derrida, Séminaire La peine de mort, I, p. 167.

25. Ibid., p. 168. 
désœuvrement: "Sans la mort, tout s'effondrerait dans l'absurde et dans le néant ${ }^{26}$. " Derrida a donc raison de rappeler à quel point Blanchot « reproduit $[\ldots]$ sans constituer une thèse explicite en faveur de la peine de mort [...] le noyau argumentatif, le philosophème classique de toutes les grandes philosophies du droit favorables à la peine de $\operatorname{mort}^{27}$ ». S'inscrivant (à cette époque, du moins) dans la lignée de La phénoménologie de l'esprit (par le biais des analyses d'Alexandre Kojève et de Georges Bataille), Blanchot semble effectivement penser la dignité humaine de concert avec une mort non pas subie mais désirée, à l'image d'un certain «amor fati» préconisé par Nietzsche. Évoquant le cas de Kant en particulier, Derrida souligne la continuité entre le discours de Blanchot et celui de ses prédécesseurs germaniques:

Un droit qui renoncerait à inscrire en lui la peine de mort ne serait pas un droit, ce ne serait pas un droit humain, ce ne serait pas un droit digne de la dignité humaine ", [car] "le droit implique que quelque chose vaut plus que la vie et que donc la vie doit ne pas être sacrée comme telle, elle doit pouvoir être sacrifiée pour qu'il y ait du droit ${ }^{28}$.

Si l'on suit ce fil argumentatif jusque dans ses dernières conséquences, la vie n'a pas de valeur en elle-même et pour elle-même, étant fatalement suspendue à sa négation, fût-elle symbolique ou littéraire, au nom de quelque chose qui lui est supérieur, ce qui a pour effet de reconduire le complexe théologico-métaphysique que Derrida tente de déconstruire tout au long du Séminaire La peine de mort.

Le droit prend la mort pour objet non moins que la vie, peut-être même davantage. Il s'agit plus exactement ici du «droit de se tuer, d'être tué ou de tuer: d'accéder à la mort en excédant la vie naturelle ou la vie biologique ou dite animale ${ }^{29}$ ", autant d'actes qui vont de pair avec une certaine liberté de type existentialiste, alors dans l'air du temps. Ici encore, Derrida reste sur ses gardes une fois parvenu au cœur ${ }^{30}$ de ce que l'argumentation blanchotienne a de plus terrible. Peut-être s'excuse-t-il même devant les affirmations volontiers excessives de son ami, nous rappelant qu'il y a dans «La littérature et le droit à la mort » «encore autre chose, qui pourrait faire basculer, jusque dans son contraire, l'analyse de cette essence de la littérature comme terreur ${ }^{31}$ ». Après tout, la section consacrée à Sade et à la Révolution française, tout en étant celle vers laquelle le titre fait signe, lui

26. Blanchot, «La littérature et le droit à la mort », p. 313. Les italiques sont dans le texte. 27. Derrida, Séminaire La peine de mort, I, p. 170.

28. Ibid.

29. Ibid., p. 171.

30. Ce mot, en apparence des plus anodins, occupe une place vitale dans l'argumentation derridienne, ainsi que le souligne Peggy Kamuf dans "At the Heart of the Death Penalty ", Oxford Literary Review, vol. 35, n 2, 2012, p. 241-251.

31. Derrida, Séminaire La peine de mort, I, p. 172. 
assurant ainsi une certaine préséance, n'est qu'un moment parmi d'autres dans une longue réflexion sur la chose littéraire, qui doit finalement plus à Mallarmé qu'à toute autre figure tutélaire, Hegel (ou plutôt Hegel-KojèveBataille) y compris. C'est ce que Derrida tient à faire valoir au moment de clore cet unique développement du Séminaire La peine de mort consacré à Blanchot, en attirant notre attention sur ce qu'il appelle les "trois motifs" qui sous-tendent «La littérature et le droit à la mort » : «1. Le langage littéraire est contradictoire ${ }^{32} » ;$ "2. [...] le principe de mort [...] est aussi un principe de résurrection et de salut ${ }^{33} » ;$ « 3 . [...] Blanchot ne parle du mourir que comme d'une impossibilité ${ }^{4}$ ", trois motifs que l'on pourrait résumer en rappelant qu'une proposition blanchotienne typique telle que "la vie, c'est la mort » est à la fois littéraire du fait de son caractère auto-contradictoire et une vérification de la structure de ce qu'il appelle le mourir (lorsque la vie cesse, la mort prend fin aussi - seule demeure alors son impossibilité, que Thomas l'Obscur décrit comme la résurrection de la mort même ${ }^{35}$ ).

Autrement dit, il n'y aurait pas à proprement parler de discours sur la peine de mort dans «La littérature et le droit à la mort », à moins qu'on n'y décèle un dis-cours, c'est-à-dire un clivage délibérément amphibologique qui use des ressources de la littérature pour élever la peine de mort au rang d'absolu — d'absolu fantôme ou d'absolu sans absolu, pour parler comme Blanchot - et non l'inverse. En ce sens, le contraste entre Blanchot et Hugo est moins flagrant qu'il n'y paraît, puisque dans un cas comme dans l'autre le dernier mot revient à la chose littéraire. Même la négation hégélianomallarméenne de la vie par le langage reste un geste impuissant, fictif, à mille lieues du tranchant des textes de loi, qui ont vocation à être appliqués, pour ne rien dire de la guillotine elle-même. En clair, la mort n'a pas lieu au sein de l'espace littéraire (parce que tout y est déjà mort, selon Blanchot, ou parce que la littérature se situe toujours du côté de la vie, selon Hugo). Malgré le trait d'union esquissé par "La littérature et le droit à la mort» entre le temps révolutionnaire et le désœuvrement du temps littéraire, qui est en fait "la fascination de l'absence de temps ${ }^{36}$ ", l'anonymat, l'interruption et l'équivoque importent davantage, en dernière analyse, que l'appareil étatique révolutionnaire qui met en œuvre la peine de mort, ainsi que le confirmera, vingt ans plus tard, l'aventure de Mai 68, où le risque d'exécution ne sera qu'une arrière-pensée.

Cette auto-contradiction, équivocité ou ambiguïté, si essentielle pour comprendre la mort et la littérature chez Blanchot, annonce à bien des

32. Ibid.

33. Ibid.

34. Ibid., p. 174.

35. «Il marchait, seul Lazare véritable dont la mort même était ressuscitée» (Blanchot, Thomas l'Obscur, nouvelle version, Paris, Gallimard, 1992, p. 42.)

36. Blanchot, L'Espace littéraire, Paris, Gallimard, 1988, p. 25. 
égards le neutre, qui a pour effet de neutraliser le couplage mort/vie tout en étant plus inséparable que jamais de cette injonction à "survivre» sur laquelle Derrida porta très tôt son attention. Proche à plus d'un titre du phármakon et de l'indécidable, le neutre n'est pas simplement synonyme de la neutralité, tout comme l'indécidabilité ne saurait nous acquitter de la décision et/ou de l'éthique. Au contraire, le neutre implique la suspension — et seulement cela — du tranchant de la décision, l'arrêt de l'arrêt de mort, ainsi que Derrida le rappelle sans discontinuer. Il dérègle la dialectique hégélienne et, partant, détraque la mécanique de la décapitation et de la Terreur, sans épargner la part la plus effrayante et la plus menaçante du dispositif argumentatif de «La littérature et le droit à la mort»; il fait signe vers la mort, voire l'appelle de ses vœux, mais sans jamais pouvoir l'accomplir comme telle. Une fois neutralisée, la mort est donc cette guillotine dont le couperet serait à jamais arrêté dans le vide, entre la traverse et le cou du condamné, l'arrêt qui arrête l'arrêt, pour revenir à L'Arrêt de mort.

Telle serait la loi de l'espace littéraire. Et si le Séminaire La peine de mort n'évoque pas L'Arrêt de mort en particulier, "Survivre» aura déjà fourni une lecture exemplaire du récit de Blanchot, qui tente de relater l'œuvre neutralisante de la mort. Mieux, "Survivre » aura déjà fait valoir la dimension légale de l'arrêt de mort, d'où son statut d'intertexte privilégié relativement au Séminaire La peine de mort, comme en témoigne ce passage qui mérite d'être cité in extenso:

Un arrêt en français, intervient au terme d'un procès, la cause ayant été plaidée, pour juger. Le jugement faisant arrêt vient clore l'affaire, il rend une décision de justice. C'est une sentence. Un arrêt de mort est une sentence qui condamne à mort. Il s'agit donc bien d'une chose comme cause et d'une décision quant à la chose. La Chose se trouve être, comme dans le texte de Blanchot, la Mort; et la décision de mort concerne la mort, cause et fin. Pas plus que la chose, la mort ne vient naturellement. Elle a un rapport obscur à la décision, plus précisément à quelque sentence, à quelque langage qui fait acte, à la fois action et archive, en laissant trace. L'arrêt de mort fait de la mort une décision ${ }^{37}$.

Je me contenterai ici de quelques brèves remarques: la "Chose», entendue comme cet étant singulier et innommable qui se dérobe à toute tentative de saisie, est également pourvue d'un sens juridique. Du fait de son indétermination apparente, elle nécessite que l'on prenne une décision à son sujet, que l'on tranche une cause. Dans le texte de Blanchot, c'est la mort en tant que telle qui se voit traduite en justice, mettant en abyme le théâtre ${ }^{38}$ de la peine

37. Derrida, «Survivre», p. 154-155.

38. Le Séminaire La peine de mort ne cesse de faire valoir le caractère proprement spectaculaire, dramaturgique et oculaire de la peine capitale. Pour une analyse de cette question, $c f$. Elizabeth Rottenberg, "A New Primal Scene: Derrida and the Scene of Execution », in Kelly Oliver et Stephanie Straub (dir.), Deconstructing the Death Penalty: Derrida's Seminars and the New Abolitionism, New York, Fordham University Press, 2018, p. 32-62. 
de mort dans son ensemble à travers la chose littéraire. Par ailleurs, cette Chose, tout comme le neutre dont elle dépend, est l'inhumanité même, l'anonymat comme négation de l' "homme ", ce qui veut dire en un sens que personne ne meurt dans L'Arrêt de mort — nous sommes donc loin ici de la pensée heideggérienne selon laquelle le Dasein serait un être-pour-la-mort. Encore une fois, c'est l'impossibilité de la mort qui se vérifie à travers ce schéma. Voilà sans doute pourquoi J., la jeune femme dont nous lisons progressivement la mort dans la première partie de L'Arrêt de mort, demande à l'infirmière si elle a "déjà vu la mort ". Lorsque celle-ci lui répond qu'elle a seulement "vu des gens morts", J. rétorque: «Eh bien, vous la verrez bientôt $»^{39}$.

Davantage que les mourants (les vivants) qui la subissent, c'est donc la mort en tant que cause ou chose - peut-être même en tant que chose en soi - qui fait l'objet d'une décision ici, nous rappelant que pour Derrida non moins que pour l'auteur de La Folie du jour (voire Kafka, cet autre penseur du procès de la mort, dont la figure de Gracchus a beaucoup marqué la thanatologie blanchotienne), rien ne se saurait se soustraire au jugement. Mais si ce jugement a vocation à être juste (en termes de justice aussi bien que de justesse), il ne saurait l'être de manière irréfutable, car «de la mort, nul n'a de savoir ${ }^{40}$ ", comme le dit bien la formule de Socrate, citée (et traduite) par Blanchot dans «Le dernier à parler ». C'est là une ignorance proverbiale qui va de pair avec la chose, justement, en ce que celle-ci ne cesse de se retirer et de demeurer en deçà d'un savoir accaparant et conceptualisant, en deçà de l'espace dit «humain". En effet, on désigne du nom de «chose» ce lieu où il n'y a plus de nom qui tienne (hormis le plus vague et le plus insignifiant: celui de "chose»), où quelque chose tient lieu d'autre chose et ainsi de suite, à l'infini.

Que signifie «mort» dans le syntagme «l'arrêt de mort »? Qu'est-elle, cette chose qui est la mort, qui donne la mort? Poser la question, tout comme le fera le Séminaire La peine de mort dans son ensemble, c'est ouvrir le langage à la béance de l'abîme, à la littérature. Lorsqu'il évoque, dans L'Espace littéraire, le cas de Kirillov, ce personnage des Démons de Dostoïevski (mieux connu à l'époque sous le titre Les Possédés) qui souhaite devenir immortel en se suicidant, Blanchot affirme que l'"[o]n ne peut "projeter" de se tuer. Cet apparent projet s'élance vers quelque chose qui n'est jamais atteint, vers un but qui ne peut être visé, et la fin est ce que je ne saurais prendre pour fin ${ }^{41}$ » avant de préciser plus loin que quiconque «veut mourir, ne meurt pas, perd la volonté de mourir, entre dans la fascination nocturne où il meurt

39. Blanchot, L'Arrêt de mort, Paris, Gallimard, 1977, p. 30.

40. Blanchot, "Le dernier à parler", dans Une voix venue d'ailleurs, Paris, Gallimard, 2002, p. 71.

41. Blanchot, L’Espace littéraire, p. 129. 
dans une passion sans volonté ${ }^{42}$. Comme toujours lorsqu'il est question du mourir chez Blanchot, c'est la passivité qui prime, c'est-à-dire cette absence de volonté qui empêche quiconque - hormis la mort, pour peu qu'on la pense comme cette chose qui appelle à elle tous les noms - de donner la mort, de faire de la mort quelque chose que je donne. Seul on (se) donne la mort; il n'y a là personne.

On pressent les conséquences potentiellement scandaleuses que cela peut avoir pour la peine de mort, ainsi que pour la question de l'euthanasie: ne risque-t-on pas en effet de blanchir des instances meurtrières au nom de l'impersonnalité ? Cette question des plus violentes s'impose à son tour dans la pensée derridienne, depuis Le parjure et le pardon et au-delà. Quoi qu'il en soit, le flou, voire l'irresponsabilité qu'implique le "on» neutre de la mort a surtout une incidence sur le plan du langage ici, autorisant Blanchot à donner le titre d'Arrêt de mort à un livre qui, selon toute vraisemblance, relate une scène d'euthanasie plutôt qu'une condamnation à mort juridique ou même fatidique. Cela dit, le mot d' "euthanasie " pose lui aussi un problème: cette mort prétendument "bonne", qui n'avait pas de connotations médicales avant que Francis Bacon ne déploie à nouveau le terme au $\mathrm{XVII}^{\mathrm{e}}$ siècle, est en soi un concept des plus clivants, jusque dans ces pays qui, à l'image du Canada, l'ont légalisée. On parle ainsi, dans les textes officiels, d' "aide médicale à mourir ", ce qui a pour effet de substituer aux connotations morales, voire métaphysiques de l'euthanasie un langage plus humaniste et scientifique, pour ne pas dire positiviste, qui signifie qu'une telle mort n'est jugée bonne qu'à partir du moment où elle est prise en charge par le corps médical. Ce faisant, on évacue la notion de suicide assisté, sans doute trop peu euphémique, même si la chose, elle, peut se pratiquer en toute légalité à partir du moment où l'on estime, toujours à titre exceptionnel, que la mort est devenue préférable à la vie, que la cause a été tranchée dans ce sens plutôt que dans l'autre. Enfin, il est à noter que ces déclinaisons relèvent toutes d'un certain "droit de mourir " qui fait écho au droit à la mort proposé par Blanchot puis analysé par Derrida.

En amont de la médecine et à l'image de la peine de mort, l'euthanasie suppose ainsi une décision juridique, puisque la loi exige que l'on soit en mesure de déterminer si la mort du souffrant sera, en dernière analyse, une «bonne " chose. Il n'y a jusqu'au suicide comme tel qui ne se soustraie pas entièrement à l'État: bon nombre de pays l'interdisent, tels que la Guyane, le Bangladesh, la Jordanie, le Liban, l'Arabie saoudite et l'Uruguay (dans le cas de ce dernier, il est toutefois possible de se faire pardonner à titre posthume). Et pourtant, de toutes ces nations, seul l'Uruguay a aboli la peine de mort, ce qui laisse entendre qu'il y a une complicité entre l'État qui condamne à mort et celui qui cherche à garder la vie sauve, un véritable droit de vie et 
de mort. A contrario, les États qui autorisent aujourd'hui l'euthanasie ${ }^{43}$ sont pour l'essentiel des héritiers du libéralisme, voire de traditions protestantes plus enclines à valoriser la responsabilité individuelle: ils envisagent la mort comme un choix personnel et librement consenti. Ainsi, le sujet — défini ne serait-ce que partiellement, lointainement, en tant qu'être-pour-la-mort choisit par lui-même et pour lui-même l'heure et la modalité (du moins jusqu'à un certain point) de sa mort à venir. Dans ce schéma intentionnaliste, le sujet a donc une consistance qui s'inscrit dans la durée, étant conçu comme suffisamment stable et égal à lui-même pour assumer une telle responsabilité. Il importe enfin de souligner qu'à la différence de la logique spectaculaire de la peine de mort et malgré l'implication de l'État dans cet événement mortuaire, l'euthanasie suppose un espace privé, souvent familial, parfois solitaire, où le sujet mourant peut jouir, une dernière fois, du libre-arbitre qui lui serait propre, à l'écart de la société, quoique selon des termes préalablement établis par la pólis. La distance entre de tels cadres législatifs et le neutre de la mort est infinie.

Le paradoxe n'est pas anodin: l'État de droit, tout en reconnaissant l'existence d'un sujet digne de ce nom (la dignité est d'ailleurs l'un des motsclés de l'euthanasie, comme en témoigne emblématiquement l'association suisse Dignitas), cherche à imposer sa souveraineté même et surtout là où il y a exception: c'est, on le sait, une des problématiques, empruntées à Carl Schmitt, qui retiendra longuement l'attention de Derrida dans les années 1990, y compris dans le Séminaire La peine de mort. Rien ne doit échapper à l'aire d'influence du ou des souverains, car toute chose - y compris ce que la théologie appelle «l'au-delà » — se transmue pour lui en une cause nécessitant un jugement final, un arrêté qui ne sait pas s'arrêter, c'est-à-dire un geste juridico-critique qui ne recule pas devant la mort, voire qui préfère cette cause suprême et suprêmement mystérieuse à toute autre, suivant un principe fascinateur qui est à la fois proche de l'espace littéraire, où la venue de la fin est à l'origine de toute fascination, et infiniment lointain, puisque la littérature est aussi ce domaine où l'on n'en finit plus de finir, où l'arrêté n'advient jamais, où l'arrêt s'arrête.

Nous l'avons vu: de telles affirmations, qui frôlent le régime littéraire, voire poétique, sont indissociables d'un idiome précis, en l'occurrence de la langue française, qui tente, tant bien que mal, comme toute langue, de trouver un idiome pour dire ce qui est en jeu ici. La deuxième partie de L'Arrêt de mort nous donne à lire, entre les lignes, cette question à laquelle le Séminaire La peine de mort ne cesse de faire signe.

Après le décès de J., le narrateur évoque le bombardement de Paris pendant la Seconde Guerre mondiale. Face à la menace alors en cours, il est contraint de s'abriter dans le métro aux côtés d'une autre de ses nombreuses

43. Tous ont aboli la peine de mort. On notera cependant qu'aux États-Unis, les lois varient drastiquement d'un État à l'autre. 
fréquentations féminines, une dénommée $\mathrm{N}$. (on notera au passage le recours quasi systématique aux initiales, censées garantir l'anonymat de personnages pourtant fictifs). Mais au lieu de s'appesantir, comme on s'y attendrait, sur la gravité de la situation, il note que «[c]es cérémonies alors manquaient de sérieux. Et N. trouvait agréable n'importe quelle raison de

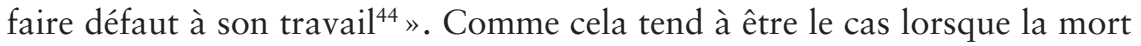
est proche dans les écrits de Blanchot - et l'on pense notamment à L'Instant de ma mort, dont on connaît l'importance chez Derrida —, c'est le registre de la légèreté qui l'emporte, légèreté qui se traduit ici par le jeu des langues, de leur pluralité et de leur étrangeté:

Depuis quelque temps, je lui parlais dans sa langue maternelle, que je trouvais d'autant plus émouvante que j'en connaissais moins les mots. Pour elle, elle ne la parlait pour ainsi dire jamais, du moins avec moi, et cependant, si je commençais à ânonner, à lier ensemble des termes maladroits, à former des locutions impossibles, elle les écoutait avec une sorte de gaieté, de jeunesse, et à son tour elle me répondait en français, mais un français différent du sien, plus enfantin et plus bavard, comme si la parole fût devenue irresponsable, à la suite de la mienne, employant une langue inconnue ${ }^{45}$.

Au milieu de cette foule qui s'amasse sous terre dans l'espoir de se soustraire à la mort, chacun est rendu à l' "anonymat " et au "désœuvrement ", termes dont on sait l'importance pour Blanchot et qui vont de pair avec une certaine idée de la littérature comme interruption, comme glissement d'un idiome à l'autre, comme devenir-étrange ou étranger de la langue. La parole devient ainsi «irresponsable », ce qui veut dire qu'elle ne répond plus de rien et encore moins d'une quelconque loi étatique. Il devient alors possible de dire n'importe quoi, de tout dire, voire de mal dire, dans toutes les langues - elles sont désormais déliées par la mort.

La phrase (la sentence) signifie alors l'exact contraire de la death sentence: elle est une forme de survivance, d'excès, de hiatus; elle est de trop par rapport à la mort conçue comme fin ou arrêt. Et pourtant, nous l'avons vu, pour Blanchot, toute parole - à plus forte raison lorsque celle-ci est de type poétique ou littéraire - témoigne de la mort, mort qui est toujours tout autre (avec ou sans majuscules) tout en étant à ce point proche de nous «que, pensant, nous mourons ${ }^{46}$ », comme il le dira plus tard dans Le Pas au-delà. Or cette pensée est paradoxalement une pensée du dehors: bien qu'elle soit exposée à tout ce qu'elle n'est pas, à commencer par le discours babillant de la pólis, elle demeure foncièrement étrangère à ses jugements et arraisonnements. Autrement dit, si «[1]'État doit et veut voir mourir le condamné ${ }^{47}$ ", ainsi que Derrida le souligne dès la séance d'introduction du

44. Blanchot, L'Arrêt de mort, p. 99.

45. Ibid., p. 99-100.

46. Blanchot, Le Pas au-delà, Paris, Gallimard, 1973, p. 7.

47. Derrida, Séminaire La peine de mort, I, p. 25. 
Séminaire La peine de mort, la mort en tant que telle, en tant que chose en soi, en tant que non-peine ou non-condamnation, n'a nul besoin de voir quoi que ce soit, étant aveugle (davantage que la justice proverbiale). La mort a lieu, encore et toujours, et sa loi inhumaine, s'il y en a, est à peine vérifiable, à peine codifiable, à peine décidable. C'est justement pour cela qu'elle exige de "dire et penser ce qu'on peut à peine dire et penser ${ }^{48}$ ", formule qui peut d'ailleurs servir de définition provisoire de la déconstruction aussi bien que de la littérature.

Si la mort ne cesse d'appeler la parole, la parole, elle, peine à nommer la mort, ce qui ouvre sur (au moins) deux voies: celle de la littérature, qui ressasse cette peine et cette passivité à l'infini, et celle du langage efficace, qui tente d'atteindre la mort en la donnant. Elle prend alors une décision quant à la chose qui va parfois jusqu'à la scission du cap ou du chef: de la tête, donc, y compris pour que celle-ci cesse de parler. En clair, la sentence veut trancher quant à l'insaisissable, comme s'il suffisait de saisir le problème par le cou pour le régler une bonne fois pour toutes, tandis que la sentence, la phrase - ou mieux: le phrasé - cherche à se faire le témoin de cette indécidabilité, d'en finir à l'infini. Plutôt que la finalité présomptueuse de la peine de mort, c'est l'infinitude et la passivité de ce que Blanchot appelle le «mourir» qui s'affirment ici, entre ou hors les langues.

Mais ne faut-il pas en finir malgré tout? Ne faut-il pas mourir - d'une bonne mort, de préférence? Dès l'incipit, le narrateur de L'Arrêt de mort nous fait cet aveu: "Si j'ai écrit des livres, c'est que j’ai espéré par des livres mettre fin à tout cela. » Il précise ensuite qu'il "espère en finir bientôt » ${ }^{49}$. La finalité de la littérature consisterait par conséquent à mettre fin... mais à quoi, au juste? Peu importe, serait-on tenté de dire, l'essentiel est de mettre fin à quelque chose sans tout à fait y parvenir et, à travers cette opération incertaine et faillible, de faire apparaître le mouvement même du mourir, par lequel la fin des fins ne cesse d'arriver. Car le lecteur de L'Arrêt de mort ne saura jamais tout à fait quel est le «tout cela » auquel il s'agit de mettre fin, à moins que ce ne soit, comme le suggère Derrida, la mort en tant que telle, en tant que Chose ou chose en soi. On apprend même que le narrateur a déjà tenté de «donner une forme écrite » aux "événements » ${ }^{50}$ qui lui sont arrivés avant de détruire le manuscrit, ce qui signifie en un sens que la fin a déjà eu lieu, mais qu'elle est encore à venir. Comme ce sera à nouveau le cas dans L'Instant de ma mort, le texte est toujours hanté par son double, par une image antérieure à laquelle on n'aura jamais pleinement accès et qui fut déjà une reconstitution imparfaite des événements que le récit est censé relater (à moins que celui-ci ne soit «l'événement même ${ }^{51}$ »).

48. Derrida, Séminaire La peine de mort, II, p. 55.

49. Blanchot, L'Arrêt de mort, p. 7.

50. Ibid.

51. Blanchot, Le Livre à venir, Paris, Gallimard, 1986, p. 14. 
Ainsi, à peine parvenu à la fin du tout premier paragraphe, le lecteur de L'Arrêt de mort est happé par une temporalité des plus floues. Ce qui est raconté, ce n'est pas tout à fait ce qui a eu lieu, mais cela même: que c'est raconté. Et dans la mesure où le récit ne renvoie pas à une date précise pas plus précise que "octobre 1938 ", plus ou moins —, il semble n'avoir de temps que celui de son propre déroulement, une phrase à la fois. À titre d'exemple, le narrateur écrit qu'en «ce matin qui est le 8 octobre (je viens de le constater à ma surprise) et qui, par conséquent, marque à peu près l'anniversaire de la première de ces journées, je suis presque sûr que les paroles, qui ne devraient pas être écrites, seront écrites. Depuis plusieurs mois, il me semble que j'y suis résolu ${ }^{52}$.» On remarquera ainsi la surprise du protagoniste lorsqu'il s'aperçoit de la date, l'emploi de la locution "à peu près ", de l'adverbe «presque» et du verbe «sembler» — rien, absolument rien, n'est certain icis3.

D'où l'hypothèse suivante, en guise de contraste: la peine de mort, la sentence mortuaire, c'est un rapport de certitude au temps, un calendrier tranchant. Tu mourras - cela, tout un chacun le sait - à telle ou telle date - cela aussi, nous le savons, et chaque année nous survivons sans le savoir à l'anniversaire de notre mort à venir -, mais tu en connaîtras d'avance le temps et le lieu. Tandis que

[l']indétermination est un trait essentiel de mon rapport à la mort $[\ldots]$ le condamné à mort, et voilà la différence, il peut savoir, croire savoir, et en tout cas d'autres savent pour lui, en principe, en droit, quel jour, à quelle heure, voire à quel instant la mort tombera sur lui. [...] Ce savoir, cette maitrise du temps de vie et de mort, ce savoir maîtrisant et calculant du temps de vie du sujet est présupposé, je dis bien présupposé, allégué, présumé dans le concept même de la peine de mort ${ }^{54}$.

L'État décide de l'instant de la mort du condamné, de l'année, du mois, de la semaine, du jour, de l'heure et du lieu. Tel est le propre de la peine ou poiné: elle est mesurable, quantifiable, calculable, à l'instar du temps même de son avoir-lieu, qu'elle a le droit de prévoir afin de voir la mise à mort, d'en vérifier la mise en œuvre. Bien entendu, ce n'est pas de cette mort-là qu'il est directement question dans L'Arrêt de mort. S'il y a condamnation, celle-ci est plutôt d'ordre fatidique ou médical. Il importe d'ailleurs de le rappeler: un condamné, ce n'est pas seulement quelqu'un qui subit ou s'apprête à subir le châtiment de la loi (humaine, divine ou autre encore, peu

52. Blanchot, L'Arrêt de mort, p. 8.

53. Plusieurs commentateurs, à commencer par Christophe Bident (Maurice Blanchot: partenaire invisible, Seyssel, Champ Vallon, 1998), Leslie Hill (Blanchot: Extreme Contemporary, Londres et New York, Routledge, 1997) et Ginette Michaud (Tenir au secret), ont souligné la datation délibérément tremblante de ce texte, qui annonce L'Instant de ma mort. Il n'y a pas de calendrier réel qui puisse marquer le passage de ces "événements ».

54. Derrida, Séminaire La peine de mort, I, p. 301. 
importe), c'est aussi un être vivant qui souffre d'une maladie mortelle, dont les jours sont comptés, comme on dit, sans que l'on sache toutefois avec certitude quand l'instant fatal arrivera. On le sait plus ou moins, acculés comme nous le sommes à cette temporalité lacunaire et ambiguë qui s'expose dès l'incipit de L'Arrêt de mort et qui n'a rien à voir avec l'arrêt de mort au sens propre, juridique de cette expression, se rapprochant plutôt de ce que Marguerite Duras appelle «la maladie de la mort ${ }^{55}$ ».

Dans la première partie de L'Arrêt de mort, l'indétermination temporelle est, nous l'avons vu, des plus manifestes. Mieux, cette indétermination n'épargne pas la chronologie: "Son médecin m'avait dit d'elle qu'il la tenait pour morte depuis 1936 . Il est vrai que ce même médecin, qui m’a soigné quelque temps, m'a dit aussi un jour: "Comme vous devriez être mort depuis deux ans, tout ce qui vous reste à vivre est en surnombre." Il venait de m'octroyer six mois de survie et il y a de cela sept $a s^{56}$.» On le sait, le temps de la maladie est un temps qui tend vers l'imprécis, à mille lieues du tranchant de la guillotine, et cette imprécision transparaît partout dans L'Arrêt de mort, même à travers les expressions les plus banales. Ainsi, le narrateur entend la phrase "Elle se meurt ${ }^{57}$ » à plusieurs reprises, phrase prononcée par l'infirmière et difficilement traduisible, phrase dont le présent réflexif contient en germe toute la survivance anachronique qui est interrogée ici. Même une fois que le pouls de la patiente - autre métaphore temporelle — «s'éparpilla comme du sable ${ }^{58}$ ", la mort n'a pas tout à fait achevé son œuvre, car on assiste peu après à une scène miraculeuse, lazaréenne: J. s'avère être "non seulement tout à fait vivante, mais parfaitement naturelle, gaie et presque guérie $»^{59}$. Tout cela n'en finit pas de finir.

Aussi l'arrêt ne s'arrête-t-il pas là. Au contraire, lorsque le narrateur relate, après coup, le commencement de cette journée prodigieuse, il précise que «Le réveil de J. avait eu lieu à l'aube, presque avec le soleil, et cette lumière l'avait enchantée ${ }^{60}$.» C'est alors qu'intervient la scène de l'euthanasie, censée - tout comme la peine ou l'arrêt de mort - mettre fin à l'arythmie du temps une bonne fois pour toutes:

Je pris une grosse seringue, j'y réunis deux doses de morphine et deux doses de pantopon, ce qui faisait quatre doses de stupéfiants. [...] Deux ou trois minutes plus tard, son pouls se dérégla, il frappa un coup violent, s'arrêta, puis se remit

55. Marguerite Duras, La Maladie de la mort, Paris, Minuit, 1983. Blanchot commente ce texte dans La Communauté inavouable, Paris, Minuit, 1984.

56. Blanchot, L'Arrêt de mort, p. 13-14.

57. Ibid., p. 34.

58. Ibid.

59. Ibid., p. 36.

60. Dans le Séminaire La peine de mort, Derrida nous rappelle à plusieurs reprises qu'une fois fixé le jour de l'exécution, la scène commence toujours à l'aube. 
à battre lourdement pour s'arrêter à nouveau, cela plusieurs fois, enfin il devint extrêmement rapide et minuscule, et «s'éparpilla comme du sable ${ }^{61}$.

Il y a donc des arrêts, ici, au pluriel, comme si J. était vouée à faire l'épreuve de plus d'une mort, à n'en plus finir. D'ailleurs, en relisant le récit de Blanchot, il est aisé de relever tous les signes avant-coureurs qui y sont disséminés, nous mettant au défi de déterminer l'instant précis où la mort commence: entre le protagoniste qui conseille à J. le parti du suicide et tous les moments où celle-ci qualifie le narrateur d'incarnation de la mort — mort qu'elle semble par ailleurs appeler de ses vœux - , on finit par en conclure que la mécanique narrative est elle-même une mise en scène fatale. Tout, dès le début, est en effet mobilisé en vue de ce coup de théâtre ou spectacle final, comme si l'œuvre elle-même avait orchestré cette condamnation à mort pour le plaisir cathartique du lecteur.

C'est là une sorte de parodie de la ponctualité ou scansion de la peine de mort, car L'Arrêt de mort n'est pas moins le faux-récit du temps à contretemps, du don de la mort dans toute son équivocité et indécidabilité, de l'instant où la mort brouille la frontière entre moi-même et autrui de manière décisive, où la mort et le meurtre se relaient jusqu'à se confondre. Après avoir constaté le décès de J., le narrateur ajoute d'ailleurs ces mots : "Je n'ai aucun moyen d'en écrire davantage. Je pourrais ajouter que, pendant ces instants, J. continua à me regarder avec le même regard affectueux et consentant et que ce regard dure encore, mais ce n'est malheureusement pas sûr ${ }^{62}$. » Rien n'est sûr, donc, tout comme il n'est pas sûr que la scène en principe ultérieure à celle de l'euthanasie - la scène de la résurrection, narrée plus tôt dans le récit — soit autre chose qu'un fantasme, un désir de transmuer le meurtre en moment sotériologique. Il n'est pas sûr non plus que J. ait pleinement désiré cette mort, malgré les apparences, ou que le protagoniste n’ait pas cherché à accélérer les choses pour le "plaisir» de tuer. Qui porte la responsabilité de cette mort? Ce "on » anonyme et neutre qui (se) donne la mort peut-il être pénalement responsable d'un tel instant ? L'Arrêt de mort n'a aucune réponse à apporter à de telles questions, car s'il y a une loi de la littérature, elle a vocation à demeurer indécidable.

Or ce sont les écrivains, écrit Derrida dans le Séminaire La peine de mort, qui «instituent la responsabilité et se donnent le droit sacré de faire la loi au-dessus des lois, de se faire les représentants de la justice éternelle audessus du droit, et donc de la justice divine ${ }^{63}$ ». Ils peuvent ainsi pourfendre la peine de mort au nom de la vie, à l'instar de Victor Hugo, ou montrer le neutre qui gît à même la chose morte, à l'envers de toute justice juridique ou théologique, tel Blanchot mettant en scène non seulement la question philo-

61. Blanchot, L'Arrêt de mort, p. 52. Le lecteur est donc encouragé à comparer le pouls à un sablier.

62. Ibid.

63. Derrida, Séminaire La peine de mort, I, p. 160. 
sophique de l'euthanasie mais l'impossible récit du mourir, à mi-chemin entre la mort et la résurrection, entre fiction et vérité - limite paradoxale, s'il en est. "Si vous ne me tuez pas, vous êtes un meurtrier", lance J. à son médecin, à quoi le narrateur ajoute, avec une insistance des plus troublantes sur le phrasé de la sentence: "J'ai vu, depuis, un mot analogue attribué à Kafka. Sa sœur, bien incapable de l'inventer, me l'a rapporté sous cette forme et le médecin l'a à peu près confirmé (il se rappelait qu'elle avait dit: "Si vous ne me tuez pas, vous me tuez.") ) ${ }^{64}$ En admettant que ces tournures expriment bel et bien une seule et même idée - celle d'une mort pire que la mort même, d'un meurtre plus cruel que l'acte même de tuer - , nous sommes ici au cœur aporétique du problème: la possibilité que la mort puisse s'avérer meilleure que la vie, que dans certains cas mourir vaille davantage que vivre, pour des raisons que l'État de droit ne saurait pleinement circonscrire. Or cette possibilité est elle-même rongée par une incertitude essentielle, qui menace à tout moment de basculer dans son contraire.

Il ne s'agit pas non plus de résoudre cette question à travers l'idiome analytique de la philosophie. Derrida laisse d'ailleurs la problématique en suspens, tout en s'y référant à plusieurs reprises dans le Séminaire La peine de mort. Dans le second volume, il évoque ainsi « une vie qui serait pire que la mort » et « un laisser-vivre pire que donner-la-mort » avant de préciser que cela ouvre plus exactement " au problème de l'euthanasie " ${ }^{65}$, problème dont il décline rapidement, sous forme d'anaphore, les principales facettes, sans cependant les développer plus avant. Ce n'est pas là le propos, semble-t-il nous signifier, mais près de vingt ans plus tôt, dans «Survivre ", il aura tout de même commenté la scène euthanasique de L'Arrêt de mort en écrivant que "[1]'arrêt de mort porte en lui ce double bind qui fait de toute mort un crime $^{66}$ ", affirmation qui, dans la foulée de Blanchot, rend tout à coup impossible, du moins en apparence, la distinction entre peine de mort et euthanasie, ainsi qu'entre ma mort et celle d'autrui: toutes se voient alors absorbées par une sorte de trou noir sans visage, un «on» anonyme qui ôte à l'être humain ce qu'il et ce qu'elle a prétendument de plus propre: non seulement l'être-pour-la-mort heideggérien mais aussi la peine de mort comme "propre de l'homme ${ }^{67}$ ", pour citer l'hypothèse qui inaugure le séminaire tout entier. C'est la mort comme "il y a " (au sens de Levinas) ou comme chose absolument neutre, mort qui déstabilise jusqu'à la "peine" que le Séminaire tente de circonscrire et qui hante le propos tout entier depuis les marges.

$$
* * *
$$

64. Blanchot, L'Arrêt de mort, p. 29.

65. Derrida, Séminaire La peine de mort, II, p. 37.

66. Derrida, «Survivre», p. 163.

67. Derrida, Séminaire La peine de mort, I, p. 23. 
En guise de coda et tout en soulignant à nouveau l'impossibilité de faire parler (dans tous les sens de cette expression) la question de la peine de mort dans tout ce qu'elle a d'irréductiblement multiple, j'aimerais rapidement évoquer un dernier terme, qui rôde dans les parages de cette problématique depuis le tout début: la torture. Celle-ci est en effet indissociable de la peine de mort, non seulement parce qu'elle est parfois pratiquée avant ou pendant l'exécution du condamné (l'écartèlement en offre un exemple quasi paradigmatique) mais aussi parce qu'elle joue un rôle essentiel dans l'histoire de nos attitudes (occidentales, d'abord et avant tout) à l'égard du bien-fondé ou non de la peine capitale. Derrida le rappelle dans le Séminaire La peine de mort: à partir d'un certain moment, peut-être même d'une certaine époque, la cruauté et le sang - deux concepts qui vont de pair avec la peine de mort — ne sont plus perçus d'un bon œil lorsqu'il s'agit d'appliquer le châtiment. Les discours abolitionnistes classiques misent par conséquent sur le pathos, en invoquant la cruauté du supplice et le sang qui en découle. Or de tels réquisitoires - et c'est l'une des impasses que Derrida tente de penser, voire de dépasser tout au long du séminaire ${ }^{68}$ — peuvent malheureusement se retourner contre le tribun en ce qu'ils ne tiennent pas compte de la possibilité d'adoucir ou d'atténuer l'exécution. En effet, il faut composer ici avec une volonté de raffinement, allant croissant — au même rythme que la marche de ce qu'on appelle le "progrès » — dans l'application de la peine de mort, de Joseph Ignace Guillotin jusqu'à l'injection létale telle qu'elle est aujourd'hui pratiquée aux États-Unis, tout particulièrement ${ }^{69}$.

Il s'agit donc, encore une fois, d'un problème temporel. En effet, la torture est tout entière dans la durée, dans l'interminable prolongation du supplice: elle ne s'arrête pas, au contraire de l'arrêt de mort au sens le plus courant de cette expression, arrêt censé dans certains cas apporter un répit au condamné, voire rien de moins que le salut, peut-être même celui de son âme. Le tranchant de la guillotine, tout comme l'anesthésie qui sert de prélude à l'injection létale, a donc pour visée de garantir le bien-être du condamné, comme si on pouvait par là lui donner une bonne mort, même lorsque celle-ci est «imméritée» du fait de la gravité du ou des crimes

68. L'exigence de cette pensée est infinie, ainsi que le fait remarquer Michael Naas: "If [...] the theologico-political remains the prime target of this deconstruction, then Derrida seems to be suggesting that we must add to that long list of things that call out for deconstructive analysis-sovereignty, democracy, literature, work, international law, religious tolerance, cosmopolitanism, forgiveness, the death penalty, and so on-deconstruction itself, which has now been shown to have a Judeo-Christian heritage that needs to be reread and deconstructed. In the end, then, not even deconstruction comes out unscathed in this deconstruction of the death penalty. " ( The Philosophy and Literature of the Death Penalty: Two Sides of the Same Sovereign ", The Southern Journal of Philosophy, vol. 50, 2012, p. 55.)

69. Ce qui importe surtout, c'est que le spectateur ait le sentiment d'assister à une scène d'euthanasie, car l'injection létale n'a pas toujours l'effet escompté du côté de la victime. Que les symptômes du supplice soient invisibles: voilà l'essentiel. 
commis. Derrida le dit bien: «l'instantanéisme et l'anesthésie, l'instantanéisme quasi intemporel et l'insensibilité, la non-douleur, la non-cruauté, la douceur même, sont indissociables ${ }^{70}$ ». Mieux, cette "non-cruauté absolue » (voilà du moins ce qu'elle désire être) va de pair, précise-t-il, avec «l'euthanasie $»^{71}$.

Le temps - ce que Martin Hägglund appelle, dans son livre sur Derrida, "the time of life $e^{72}$ » - se situerait ainsi du côté de l'affect, de la vie comme passion: "c'est la sensibilité ou la réceptivité, l'affection" qui seraient "en soi un châtiment " ${ }^{73}$. Dès lors, tuer le sujet souffrant équivaut à tuer le temps, nous libérant ainsi du pâtir et du subir qui seraient le propre de la vie. Par voie de contraste avec la crucifixion, volontiers dénoncée comme "primitive» et "barbare» (y compris par la plupart des partisans contemporains de la peine de mort), il y a effectivement lieu de concevoir la guillotine, pour ne rien dire des injections létales dernier cri, comme un analgésique chronocide: "La guillotine, ce n'est pas seulement un killer, c'est un painkiller [...] qui tue la douleur parce que d'une certaine façon, réduisant le temps au rien d'un instant, au rien qu'un instant ${ }^{74}$.» Il est aisé de relever ici ce que cette conception de la peine de mort - qui est, il importe de le rappeler, loin d'être la seule ou même la plus répandue dans le monde, car trop peu punitive selon certains - a en commun avec l'euthanasie : c'est, à défaut d'être une mort absolument bonne, la meilleure des morts possibles, la moins cruelle et la moins sanguinaire, mais aussi la plus "juste", c'est-à-dire aussi celle que l'on applique après délibération, au terme d'un processus juridique réglé sur les mœurs de la pólis. La mort instantanée, l'instant de la mort, est un événement qui est à peine un événement, à l'image des têtes de chou coupées de Hegel (et de Blanchot).

Cette volonté d'instantanéisme, de rassembler le temps en une seule et même présence absolue — c'est-à-dire la mort même —, ne se concrétisera jamais dans l'espace littéraire, car l'arrêt ou l'instant de la mort y est toujours autre qu'un simple Augenblick: il se raconte nécessairement après coup et sans jamais pleinement se laisser raconter, en un temps qui coupe court au couperet, comme dans L'Arrêt de mort et L'Instant de ma mort. C'est dans cette impossible spatio-temporalité que gît peut-être le secret de la survie, qui aura sous-tendu le dialogue entre Blanchot et Derrida du début jusqu'à la fin - et au-delà. Et c'est justement parce que la peine de mort rend non pas la vie mais la survie impossible, parce qu'elle est sans appel et sans avenir (son objectif étant de mettre fin à tout à-venir) qu'on ne saurait

70. Derrida, Séminaire La peine de mort, I, p. 308.

71. Ibid.

72. Martin Hägglund, Radical Atheism: Derrida and the Time of Life, Stanford, Stanford University Press, 2008.

73. Derrida, Séminaire La peine de mort, I, p. 308.

74. Ibid. 
en aucun cas la préférer. C'est parce qu'elle croit être en mesure de dissiper l'indécidable ${ }^{75}$, fût-il d'ordre littéraire ou philosophique ou autre encore, qu'elle ne pourra jamais l'emporter face à l'impossible contretemps de cette injonction, qui est peut-être au cœur de toute éthique, par-delà Blanchot et Derrida : il faut mourir, il faut survivre.

75. Kas Saghafi rappelle que "What the death penalty seeks is to incorporate the beyond-measure, the incalculable, into its calculation. However, what it claims to be able to determine, what it claims to measure - the incommensurable, the in-calculable-is an excessiveness beyond measure» ("Calculus", dans Deconstructing the Death Penalty, p. 150). 